\title{
Preparation of Microcapsules Containing Water Droplets Stabilized with Solid Powder and Application to Blowing Agent
}

\author{
Daisuke Nakamura ${ }^{1}$, Hiroshi Yokoyama ${ }^{2}$, Yoshinari Taguchi ${ }^{1}$, Masato Tanaka $^{{ }^{*}}$ \\ ${ }^{1}$ Department of Chemistry and Chemical Engineering, Niigata University, Niigata, Japan; ${ }^{2}$ Department of Management Information, \\ Niigata University of Management, Niigata, Japan. \\ Email: * $\underline{\text { tanaka@eng.niigata-u.ac.jp }}$
}

Received October 21 $1^{\text {st }}, 2013$; revised November $30^{\text {th }}, 2013$; accepted December $23^{\text {rd }}, 2013$

Copyright (C) 2014 Daisuke Nakamura et al. This is an open access article distributed under the Creative Commons Attribution License, which permits unrestricted use, distribution, and reproduction in any medium, provided the original work is properly cited. In accordance of the Creative Commons Attribution License all Copyrights @ 2014 are reserved for SCIRP and the owner of the intellectual property Daisuke Nakamura et al. All Copyright (C) 2014 are guarded by law and by SCIRP as a guardian.

\begin{abstract}
We have tried to prepare the microcapsules containing water droplets stabilized with solid powder by utilizing the $(\mathrm{W} / \mathrm{O}) / \mathrm{W}$ emulsion. The water droplets as core material were stabilized in the monomer droplets with titanium dioxide $\left(\mathrm{TiO}_{2}\right)$ as a particulate surfactant. Before adding the $\mathrm{TiO}_{2}$ powder into the monomer phase, the powder was modified with triethoxyvinylsilane to adjust the degree of hydrophobicity and to promote adhesion on the interface between the inner water phase and the monomer phase in the (W/O) emulsion. It was investigated how the degree of hydrophobicity of the $\mathrm{TiO}_{2}$ powder affected the stability of water droplets in the (W/O) emulsion and the $(\mathrm{W} / \mathrm{O})$ droplets in the $(\mathrm{W} / \mathrm{O}) / \mathrm{W}$ emulsion. Moreover, the microcapsule diameters were measured before and after the expansion operation where the water droplets microencapsulated were applied as a blowing agent. The expansion ratio was increased with increase in the stability of the water droplets and the amount of water microencapsulated.
\end{abstract}

\section{KEYWORDS}

Water Containing Microcapsules; Suspension Polymerization; Multiple Emulsion; Particulate Surfactant; Blowing Agent

\section{Introduction}

Microcapsules containing water as a core material have been applied in the various fields such as pharmacy, adhesives, agricultural medicine, cosmetics, food and so on, because water is able to dissolve various materials of inorganic and organic substances [1,2]. There are many reports [3-6] with respect to microencapsulation of water, in which water as the core material has been mainly microencapsulated by using the multiple emulsion like the $(\mathrm{W} / \mathrm{O}) / \mathrm{W}$ emulsion.

There is the suspension polymerization method as a typical method for preparing the microcapsules containing the water phase by utilizing the $(\mathrm{W} / \mathrm{O}) / \mathrm{W}$ emulsion.

In the preparation of microcapsules containing water

"Corresponding author. with the suspension polymerization method, it is important to form the stable (W/O) dispersion, where the water phase (W) is the core material and the oil phase (O) is the polymerizable monomer. For this purpose, the oil soluble surfactant species have been inevitably added and higher mechanical energy has been loaded. Then, the stability of (W/O)/W emulsion has been obtained by adding the water soluble surfactant into the continuous water phase under the optimum stirring conditions.

Tanaka et al. [7-9] have investigated the effect of solid powder on the stability of suspension polymerization of styrene, where various solid powders such as magnetite, carbon black, sulfur, cupperic oxide were added. Furthermore, they have prepared the polystyrene core/magnetite shell particles by suspension polymerization of styrene where magnetite was added into the (O/W) emulsion and 
acted as a particulate surfactant [10-13]. However, there are few reports which have investigated the effect of solid powder on the stability of water droplets in the (W/O) emulsion and prepared the microcapsules containing water as a core material. In this case, it is necessary to investigate how the amount of solid powder adhered on the surface of water droplets affects the stability of $(\mathrm{W} / \mathrm{O})$ emulsion, because the amount of solid powder adhered on the surface of water droplets is dependent on the contact angle of solid powder to the interface.

Namely, the effect of the degree of hydrophobicity of solid powder on the stability of water droplets in the (W/O) emulsion must be investigated in detail.

The purposes of this study are to investigate the stabilizing effect for the $(\mathrm{W} / \mathrm{O})$ and the $(\mathrm{W} / \mathrm{O}) / \mathrm{W}$ emulsion with the $\mathrm{TiO}_{2}$ powder modified by the coupling agent, to prepare the microcapsules containing water and to apply the microencapsulated water as a blowing agent for the preparation of expanded polystyrene.

\section{Experiment}

\subsection{Materials}

Materials used to prepare the microcapsules containing water by the suspension polymerization method are as follows.

\section{$\mathrm{TiO}_{2}$ as a particulate surfactant}

Titanium dioxide $\left(\mathrm{TiO}_{2}\right)$ with the mean diameter of 21 $\mathrm{nm}$ was added in the $(\mathrm{W} / \mathrm{O})$ emulsion. The $\mathrm{TiO}_{2}$ powder was modified with triethoxyvinylsilane of a coupling agent to give the various degree of hydrophobicity. Anhydrous toluene was used as the solvent to modify the $\mathrm{TiO}_{2}$ powder. Methanol was used to wash the $\mathrm{TiO}_{2}$ powder modified. As an oil soluble surfactant, polygrycerinester (Poem PR-100-Riken Vitamine Ltd.) was used.

\section{Preparation of Microcapsules}

Styrene monomer (st) was used to form the microcapsule shell together with trimethyrol propane triacrylate (TMPTA) of a bridging agent.

Polymerization initiator was 2-2 Azobis (2,4-diamethyl varellonitril)(V-65) which was used as received.

Polyvinyl alcohol (PVA: degree of polymerization 500) was used as a water soluble stabilizer and dissolved in the continuous water phase of distilled water.

\subsection{Modification of $\mathrm{TiO}_{2}$ Powder}

After the $\mathrm{TiO}_{2}$ powder of $10 \mathrm{~g}$ was added in anhydrous toluene of $300 \mathrm{~cm}^{3}$, this slurry was heated to $109^{\circ} \mathrm{C}$ in the oil bath. Then, triethoxyvinylsilane of a given concentration was poured to modify the $\mathrm{TiO}_{2}$ powder and the modification reaction was conducted for $5 \mathrm{~h}$ under temperature of $109^{\circ} \mathrm{C}$ and stirring of impeller speed of 200 rpm with a six bladed disc turbine impeller of diameter of $5.0 \mathrm{~cm}$. After the reaction, the $\mathrm{TiO}_{2}$ powder was washed twice with methanol and filtered out. In this modification process of the $\mathrm{TiO}_{2}$ powder, the concentration of coupling agent was changed from 0 to $0.1 \mathrm{~mol} / \mathrm{l}$-toluene.

\subsection{Preparation of Microcapsules}

Figure 1 shows the flow chart for preparing the microcapsules containing water as a core material. The $\mathrm{TiO}_{2}$ powder modified, the oil soluble surfactant and the polymerization initiator (V-65) were added in the monomer mixture of st and TMPTA beforehand. Water of given volume was added into the monomer mixture and then, agitated with the rotor-stator homogenizer to form the (W/O) emulsion for five min.

The $(\mathrm{W} / \mathrm{O})$ emulsion prepared thus was poured into the continuous water phase dissolving PVA of a given concentration under stirring of impeller speed of $250 \mathrm{rpm}$ to form the $(\mathrm{W} / \mathrm{O} / \mathrm{W})$ emulsion. Then, suspension polymerization was performed for $8 \mathrm{~h}$ under the conditions of $70^{\circ} \mathrm{C}$ and the impeller speed of $250 \mathrm{rpm}$ with the six bladed disc turbine impellers. After the suspension polymerization process, the microcapsules containing water were filtered out and washed with distilled water. In this fundamental operation, the concentration of coupling agent was mainly changed to give the $\mathrm{TiO}_{2}$ powder the various degree of hydrophobicity. Table 1 shows the experimental conditions adopted in this study.

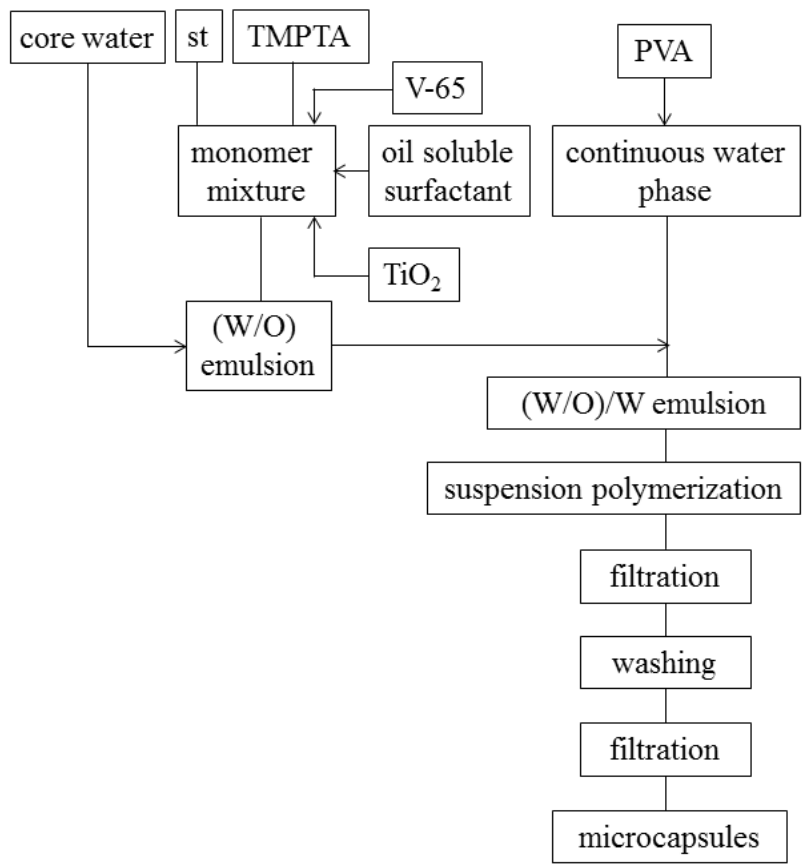

Figure 1. Schematic flow chart for preparing microcapsules containing water. 
Table 1. Experimental conditions.

\begin{tabular}{|c|c|c|c|}
\hline \multirow{2}{*}{ Continuous water phase } & Distilled water & & $300 \mathrm{~cm}^{3}$ \\
\hline & stabilizer & Polyvinylalcohol (PVA) & 0.5 wt\%-water (1.5 g) \\
\hline \multirow{5}{*}{ Dispersed phase } & Monomer & styrene(st) & $16 \mathrm{~g}$ \\
\hline & Bridging agent & Trimethanolpropane-triacrylate (TMPTA) & $8 \mathrm{~g}$ \\
\hline & Solid powder & $\mathrm{TiO}_{2}$ & $0.24 \mathrm{~g}$ (1 wt\%-monomer) \\
\hline & Initiator & V-65 & $0.66 \mathrm{~g}(0.1 \mathrm{~mol} / \mathrm{l}-\mathrm{monomer}$ mixture $)$ \\
\hline & Oil soluble surfactant & polygrycerinester (Poem PR-100) & $0.1 \mathrm{wt} \%$-monomer mixture $(0.24 \mathrm{~g})$ \\
\hline Core water phase & Distilled water & & $12 \mathrm{~cm}^{3}$ \\
\hline
\end{tabular}

\subsection{Characterization}

\section{Water content of microcapsules}

The water content $\left(F_{w}\right)$ of microcapsules prepared was calculated by Equation (1).

$$
\mathrm{F}_{\mathrm{w}}=\frac{\mathrm{W}_{\mathrm{o}}-\mathrm{W}_{t}}{\mathrm{~W}_{\mathrm{o}}}
$$

where $W_{o}$ and $W_{t}$ are the weight of microcapsules before and after drying at $60^{\circ} \mathrm{C}$ for $1 \mathrm{~h}$, respectively.

\section{Measurement of diameter distributions and mean diameters}

The diameter distributions and mean diameters (the Sauter diameter) of water droplets in the (W/O) emulsion and the microcapsules were obtained by directly measuring the diameters of about 100 water droplets and microcapsules from their microscopic photographs.

Observation of surface and cross section of microcapsules

The surface and inner structure of microcapsules were observed by scanning electron microscope (JSM-5800, JEOL Ltd.).

The dispersion features of the $\mathrm{TiO}_{2}$ powder in the matrix polymer of microcapsules were observed by electron probe microanalyzer (EPMA: XA-8900, TEOL Ltd.).

\section{Expansion ratio}

The expansion operation was conducted to investigate whether water microencapsulated acts as a blowing agent or not. Namely, the microcapsules were added into the oil bath at temperature of $130^{\circ} \mathrm{C}$ for $1 \mathrm{~h}$. Then, the diameters of microcapsules were measured from microscopic photographs. The expansion ratio was defined as Equation (2).

$$
\mathrm{E}=\frac{\mathrm{V}_{\mathrm{t}}}{\mathrm{V}_{\mathrm{o}}}
$$

where $V_{O}$ and $V_{t}$ are the volume of microcapsules before and after expansion operation.

\section{Drying rate}

The microcapsules of a given weight were dried at temperature of $50^{\circ} \mathrm{C}$ and the weight of microcapsules was measured at constant time interval during $24 \mathrm{~h}$.
From these results, the drying rate was estimated.

\section{Results and Discussion}

\subsection{Effect of Solid Powder on Stability of Multiple Emulsion}

Generally, the mechanism of the effect of solid powder on the stability of liquid droplets in Pickering emulsion is described as shown in Figure 2. Namely, it is necessary for solid powder to adhere on the surface of droplets as shown in Figure 2(a), and to protect coalescence between the droplets as shown in Figure 2(b).

If no solid powders adhere on the surface of droplets, where solid powder has to disperse in the continuous water phase (in the case of more hydrophilic solid powder) or in the oil droplet (in the case of more hydrophobic solid powder as shown in Figure 2 (c)), the droplets may coalesce each other to form the larger droplets as shown in Figure 2(c).

According to this stabilizing mechanism of solid powder, it was tried to investigate with the preliminary test whether the stabilizing effect of the $\mathrm{TiO}_{2}$ powder modified in the (W/O)/W emulsion was observed or not. These results are shown in Figure 3. Namely, first the (W/O) emulsion was formed by adding the $\mathrm{TiO}_{2}$ powder, water and oil soluble surfactant into the monomer mixture and agitating with homogenizer. Then, the (W/O)/W emulsion was formed by pouring the (W/O) emulsion into the continuous water phase as shown in Figure 3(a).

If the $\mathrm{TiO}_{2}$ powder is not modified and more hydrophilic or more hydrophobic, as no $\mathrm{TiO}_{2}$ powder adheres on the surface of water droplets and on the interface between the oil phase and the continuous water phase, the (W/O)/W emulsion has to be immediately broken. Figure 3(b) is the photograph showing the (W/O)/W emulsion broken, where the $\mathrm{TiO}_{2}$ powder and the oil droplets are dispersing in the water phase which is colored white by the $\mathrm{TiO}_{2}$ powder.

On the other hand, if the $\mathrm{TiO}_{2}$ powder is suitably modified and proper hydrophobic, as the $\mathrm{TiO}_{2}$ powder may adhere on the surface of core water droplets and on the interface between the surface of oil droplet and the 


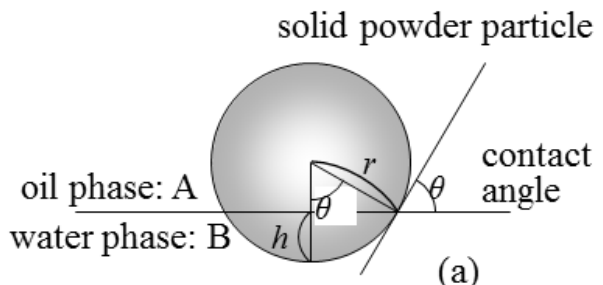

(a)
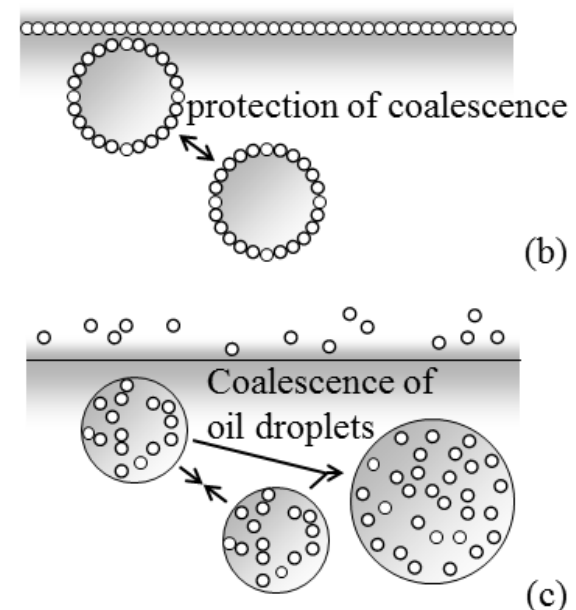

(c)

Figure 2. Stabilizing mechanism with solid powder.
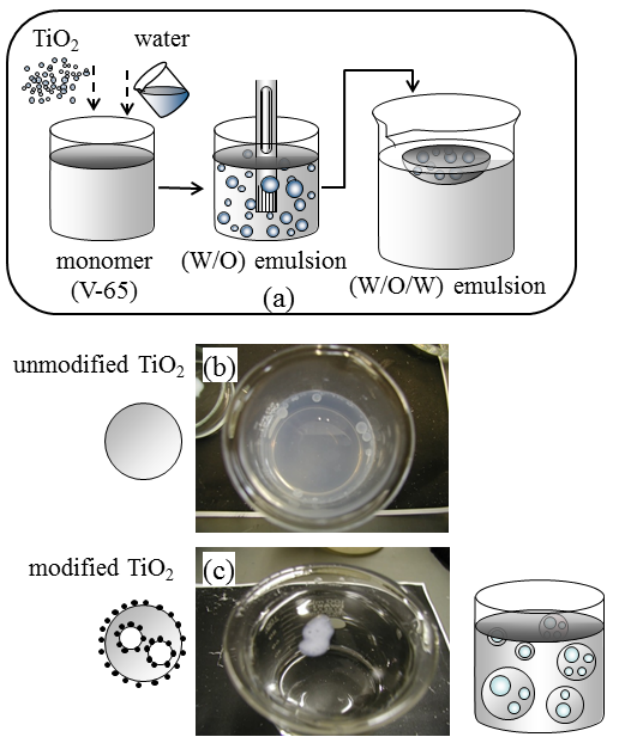

Figure 3. Effect of $\mathrm{TiO}_{2}$ powder on stability of (W/O)/W emulsion.

continuous water phase, coalescence between the water droplets and between the oil droplets has to be protected. In this case, the $(\mathrm{W} / \mathrm{O}) / \mathrm{W}$ emulsion is stabilized as shown in Figure 3(c), in which the oil droplets become white because of dispersion of core water droplets and the $\mathrm{TiO}_{2}$ powder. From these results, it is found that the $(\mathrm{W} / \mathrm{O}) / \mathrm{W}$ emulsion is able to be stabilized by the $\mathrm{TiO}_{2}$ powder with the proper hydrophobicity.

\subsection{Effect of $\mathrm{TiO}_{2}$ Powder on Stability of (W/O) Emulsion}

The effect of the $\mathrm{TiO}_{2}$ powder modified on the stability of the (W/O) emulsion was investigated by measuring the transient diameters $(\mathrm{dw})$ of core water droplets as shown in Figure 4. Namely, the core water phase was poured into the monomer mixture in which the $\mathrm{TiO}_{2}$ powder modified was dispersed to prepare the (W/O) dispersion by agitation with the rotor stator homogenizer. Then, the water droplets were sampled out and poured into the monomer mixture dissolving the oil soluble surfactant (1.0 wt\%) to prevent from coalescing. After this, the photographs of water droplets were taken as rapid as possible. From these photographs, the mean diameters of water droplets were measured as in the previous study $[14,15]$.

Figure 5 shows the transient diameters of core water droplets formed by adding the $\mathrm{TiO}_{2}$ powder modified with the coupling agent of various concentrations. It is found that the mean diameters of core water droplets increase with the elapsed time and the higher the concentration of coupling agent, the slower the diameters increase. Also, if the $\mathrm{TiO}_{2}$ powder unmodified was added, the (W/O) emulsion was immediately broken and the diameters of water droplets were not able to be measured. From these results, the more hydrophobic $\mathrm{TiO}_{2}$ powder is found to stabilize the (W/O) emulsion.

\subsection{Observation of Microcapsules}

Figure 6 shows the SEM photographs of microcapsules prepared by adding the $\mathrm{TiO}_{2}$ powder modified with the coupling agent of various concentrations.

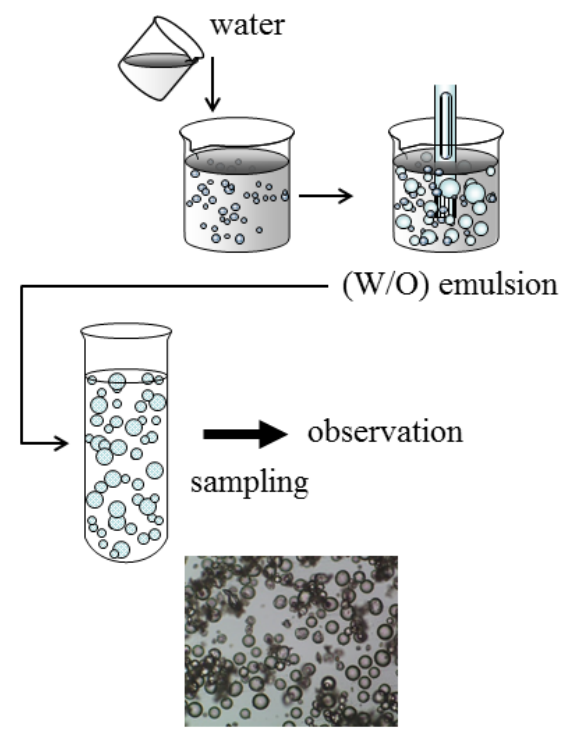

Figure 4. Observation of effect of $\mathrm{TiO}_{2}$ powder on stability of (W/O) emulsion. 


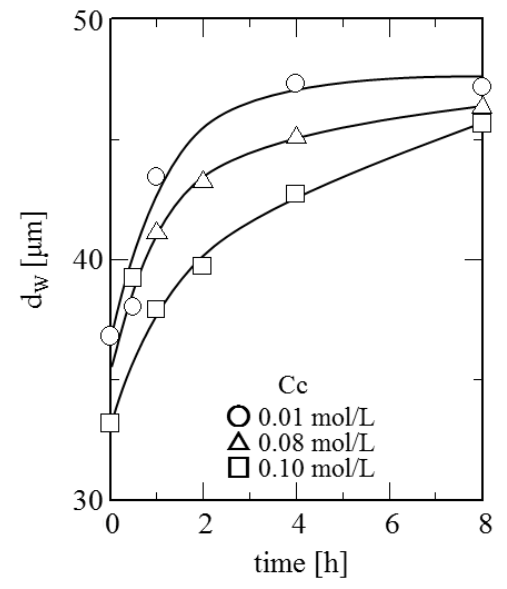

Figure 5. Transient diameters of core water droplets.

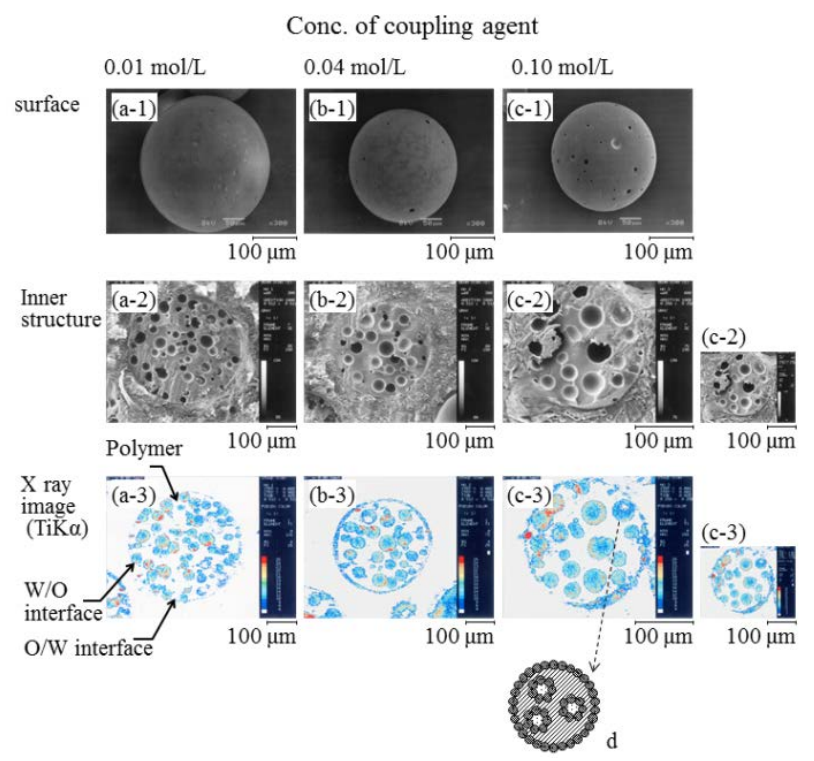

Figure 6. Observation of microcapsules.

The surfaces (a-1, b-1, c-1) of microcapsule are found to be smooth at the every preparation conditions. However, many holes, in which core water may be microencapsulated, are observed in the inner structure (a-2, b-2, c-2) of microcapsules.

From the characteristic X-ray images $(\mathrm{TiK} \alpha)$ of the inner structure (a-3, b-3, c-3), it is found that the $\mathrm{TiO}_{2}$ powder adheres on the interfaces between the core water droplet and the monomer phase and between the monomer phase and the outer water phase.

Moreover, the higher the concentration of coupling agent, the larger the $\mathrm{TiO}_{2}$ powder adhered on the interface. This phenomenon may be considered to be attributable to the fact that the $\mathrm{TiO}_{2}$ powder modified with the coupling agent of the concentration of $0.10 \mathrm{~mol} / \mathrm{l}$ may have the suitable contact angle to effectively adhere on the interface between the water phase and the monomer phase as shown in Figure 6(d).

\subsection{Effects on the Mean Diameters of Microcapsules and Core Water Droplets and the Water Content}

Figure 7 shows the effects of the concentration of coupling agent on the mean diameters of microcapsules $\left(\mathrm{d}_{\mathrm{p}}\right)$ and core water droplets $\left(\mathrm{d}_{\mathrm{w}}\right)$ and the water content $\left(\mathrm{F}_{\mathrm{w}}\right)$.

The mean diameters $\left(d_{p}\right)$ of microcapsules are found to decrease from $200 \mu \mathrm{m}$ to $70 \mu \mathrm{m}$ with the concentration of coupling agent. As the $\mathrm{TiO}_{2}$ powder comes to have the suitable contact angle to effectively adhere on the interface, the stability of both the core water droplets and the monomer droplets is increased to prevent these droplets from coalescing.

The water content $\left(\mathrm{F}_{\mathrm{w}}\right)$ slighly increases from $35 \mathrm{wt} \%$ to $38 \mathrm{wt} \%$ with the concentration of coupling agent because of increase in stability of the (W/O) emulsion.

On the other hand, as the stability of core water droplets increases with the concentration of coupling agent, the diameters of core water droplets may decrease.

\subsection{Dependence of Drying Rate on Water Content}

Figure 8 shows the dependence of the drying rate $\left(R_{d}\right)$ on the water content $\left(\mathrm{F}_{\mathrm{w}}\right)$. The drying rate increases with the water content. This may be considered to be attributable to the fact that the core water is easily released, because the shell thickness has to decrease with the water content.

However, although the water content increases with the concentration of coupling agent because of increase

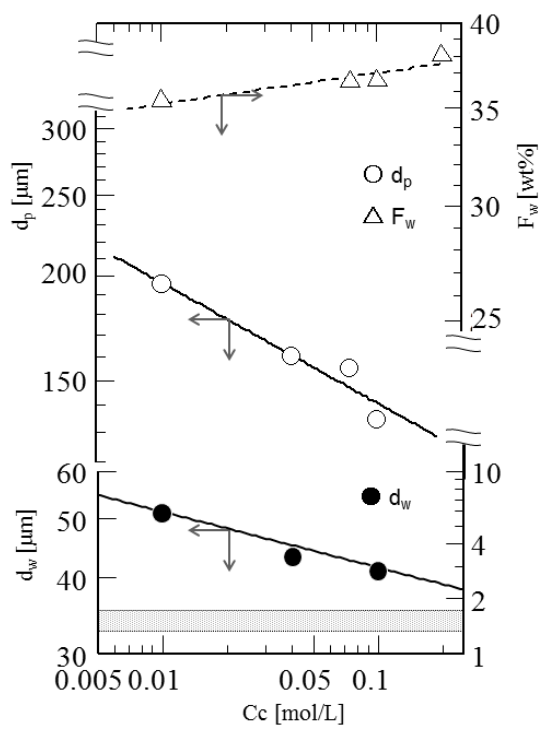

Figure 7. Effects of concentration of coupling agent on $d_{p}$, $d_{w}$ and $F_{w}$. 


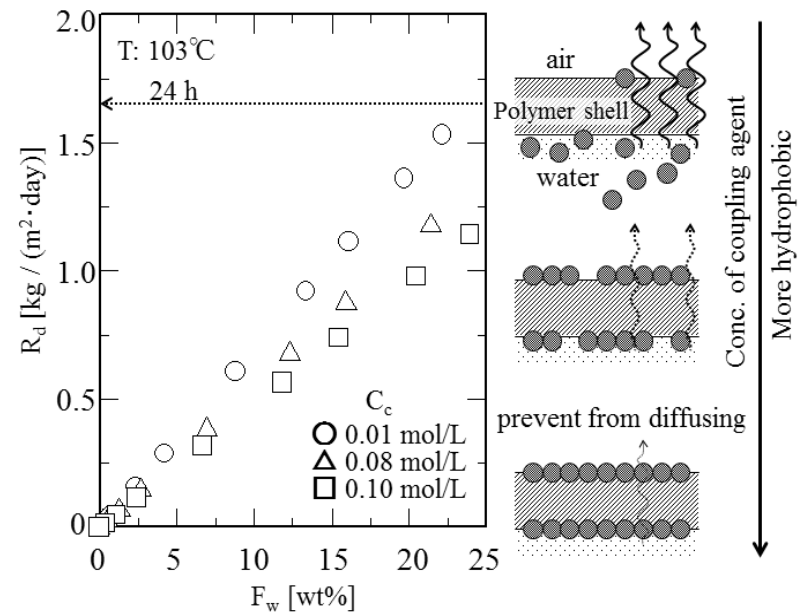

Figure 8. Dependence of drying rate on water content.

in stability of the (W/O) emulsion, the drying rate decreases with the concentration of coupling agent due to the same reason as stated just above.

This phenomenon may be attributable to the fact that as the $\mathrm{TiO}_{2}$ powder modified with the coupling agent of higher concentration comes to largely adhere on the interface, this adhesion layer composed of the more hydrophobic $\mathrm{TiO}_{2}$ powder may prevent water from diffusing through the polymer shell as shown in Figure $\mathbf{8}$.

\subsection{Application of Microencapsulated Water to Blowing Agent}

We have tried to apply the microencapsulated water to the blowing agent. Namely, the microcapsules containing water were added into the silicon oil at temperature of $130^{\circ} \mathrm{C}$ for $1 \mathrm{~h}$ as shown in Figure 9(a) and the diameters of microcapsules were measured from their photographs. Figure 9(b) shows the SEM photographs of the surface (a-1, b-1) and cross section (a-2, b-2) of microcapsules before and after expansion operation.

It is found that the diameters of microcapsules and inner holes after the expansion operation become larger than those before the expansion operation. This enlargement of diameters of microcapsules and inner holes could be considered to be attributable to expansion by the microencapsulated water.

Figure 10 shows the comparison of volume of microcapsules before and after expansion operation. The volume of microcapsules after the expansion operation is found to be 2.57 times that before expansion.

From this result, we are able to understand that the water droplets stabilized by the $\mathrm{TiO}_{2}$ powder act as a blowing agent and the $\mathrm{TiO}_{2}$ powder adhered on the interface controls the diffusion velocity of water molecule through the polymer shell.

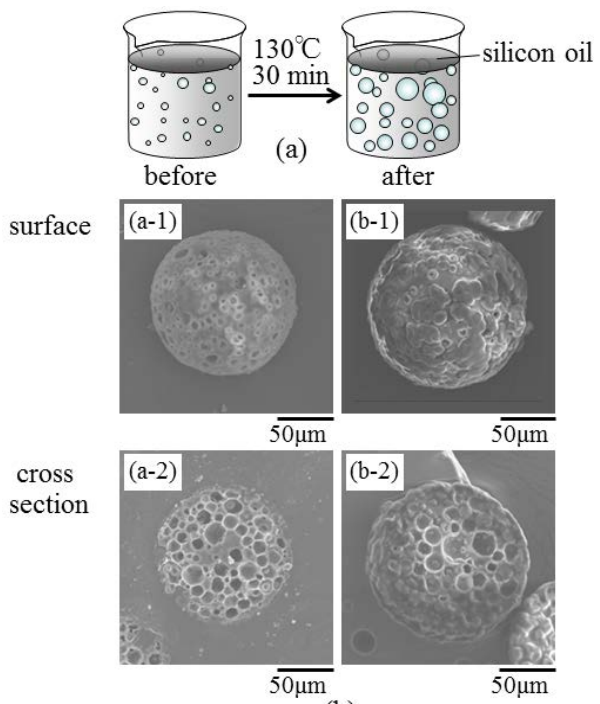

(b)

$\mathrm{Cc}=0.1 \mathrm{~mol} / 1$

Figure 9. Expansion operation and SEM photographs of microcapsule before and after expansion.

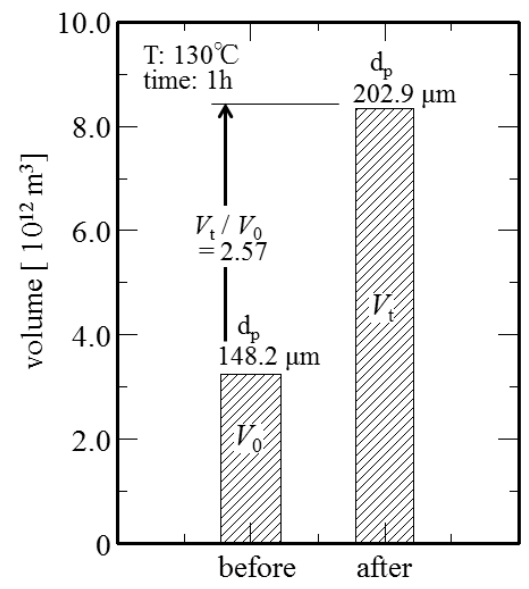

Figure 10. Comparison of volume of microcapsules before and after expansion operation.

\section{Conclusions}

We have tried to prepare the microcapsules containing water as the core material by using the stabilizing effect of the $\mathrm{TiO}_{2}$ powder in the $(\mathrm{W} / \mathrm{O})$ and the $(\mathrm{W} / \mathrm{O}) / \mathrm{W}$ emulsion and obtained the following results.

1) The microcapsules containing water were able to be prepared by the suspension polymerization method.

2) The diameters of core water droplets were decreased with the concentration of the coupling agent because of increase in stability of (W/O) dispersion.

3) The water content was increased with the degree of hydrophobicity of the $\mathrm{TiO}_{2}$ powder.

4) The microencapsulated water acted as a blowing agent. 


\section{REFERENCES}

[1] T. Kondo, "Saishin Maikurokapuseruka Gijutsu (Microencapsulation Technique) (in Japanese),” ETS, Tokyo, 1967.

[2] M. Tanaka, "Key Point of Preparation of Nano/Microcapsules,” Techno System Publishing Co. Ltd., Tokyo, 2008.

[3] J. Pallay and H. Berghmans, "Water-blown Expandable Polystyrene. Improvement of the Compatibility of the Water Carrier with the Polystyrene Matrix by In Situ Grafting Part I . Mechanism of Free Radical Grafting," Cellular Polymers, Vol. 21, No. 1, 2002, pp. 1-18.

[4] J. Pallay and H. Berghmans, "Water-blown Expandable Polystyrene. Improvement of the Compatibility of the Water Carrier with the Polystyrene Matrix by In Situ Grafting Part II. Influence of Compatibilization on the Foam Quality,” Cellular Polymers, Vol. 21, No. 1, 2002, pp. 19-33.

[5] J. Pallay, P. Kelemen, H. Berghmans and D. V. Dommelen, "Expansion of Polystyrene Using Water as the Blowing Agent," Macromolecular Materials and Engineering, Vol. 275, No. 1, 2000, pp. 18-25. http://dx.doi.org/10.1002/(SICI)1439-2054(20000201)27 5:1<18::AID-MAME18>3.0.CO;2-3

[6] J. Pallay, S. Pallayova and H. Berghmans, "Expansion of Polystyrene Using Water as the Blowing Agent,” Journal of Cellular Pastics, Vol. 43, No. 4-5, 2007, pp. 371-383. http://dx.doi.org/10.1177/0021955X07079208

[7] M. Tanaka, K. Hashimoto and K. Nakada, "Effect of Solid Powders on Stability of Emulsion," Oil Chemistry, Vol. 28, No. 7, 1979, pp. 491-496.

[8] E. O'shima and M. Tanaka, "Effect of Solid Powders on Stability of Suspension Polymerization of Styrene," $\mathrm{Ka}$ gaku Kogaku Ronbunshu, Vol. 8, No. 2, 1982, pp. 188193. http://dx.doi.org/10.1252/kakoronbunshu.8.188
[9] M. Tanaka and K. Hayashi, "Preparation of Polystyrene Particles Coated with Ferrite Powder by Suspension Polymerization,” Kagaku Kogaku Ronbunshu, Vol. 15, No. 6, 1989, pp. 1144-1152. http://dx.doi.org/10.1252/kakoronbunshu.15.1144

[10] M. Tanaka and K. Hayashi, "Preparation of Polymer Particles Covered with Ferrite Powder by Suspension Polymerization: Effect of Wettability of Powder on Composite Particle Size Distribution," Journal of Material Science, Vol. 25, 1990, pp. 987-991.

[11] M. Tanaka and K. Hayashi, "Preparation of Polystyrene Particles with Carbon Black Covered Uniformly with Ferrite Powder by Suspension Polymerization,” Journal of the Japan Society of Colour Material, Vol. 63, No. 6, 1990, pp. 321-329.

[12] M. Tanaka, A. Saito, K. Hosogai and I. Kimura, "Preparation of Fine Polymer Particles Coated Uniformly with Magnetite Powder by Suspension Polymerization,” Kagaku Kogaku Ronbunshu, Vol. 18, No. 3, 1992, pp. 330-337. http://dx.doi.org/10.1252/kakoronbunshu.18.330

[13] M. Tanaka, K. Hosogai, T. Yuda, I. Kimura and N. Saito, "Preparation of Composite Particles Composed of Polystyrene and Carbon Silicide by Suspension Polymerization," Journal of the Japan Society of Colour Material, Vol. 65, No. 8, 1992, pp. 484-491.

[14] E. O’shima and M. Tanaka, "Coalescence and Breakup of Droplets in Suspension Polymerization of Styrene,” Kagaku Kogaku Ronbunshu, Vol. 8, No. 1, 1982, pp. 86-90. http://dx.doi.org/10.1252/kakoronbunshu.8.86

[15] M. Tanaka and E. O'shima, "Effect of Viscosity of Continuous Phase on Stability of Droplets in Suspension Polymerization of Styrene," Kagaku Kogaku Ronbunshu, Vol. 8, No. 6, 1982, pp. 734-738. http://dx.doi.org/10.1252/kakoronbunshu.8.699 